\title{
Multiparameter Tests of General Relativity Using Multiband Gravitational-Wave Observations
}

\author{
Anuradha Gupta $\odot,{ }^{1,2 *}$ Sayantani Datta $\odot,{ }^{3}$ Shilpa Kastha $\odot,{ }^{4}$ Ssohrab Borhanian $\odot,{ }^{2}$ \\ K. G. Arun $\odot,,^{3,2}$ and B. S. Sathyaprakash $\oplus^{2,5,6}$ \\ ${ }^{1}$ Department of Physics and Astronomy, The University of Mississippi, Oxford, Mississippi 38677, USA \\ ${ }^{2}$ Institute for Gravitation and the Cosmos, Department of Physics, Penn State University, \\ University Park, Pennsylvania 16802, USA \\ ${ }^{3}$ Chennai Mathematical Institute, Siruseri 603103, India \\ ${ }^{4}$ Max Planck Institute for Gravitational Physics (Albert Einstein Institute), Callinstraß e 38, D-30167 Hannover, Germany \\ and Leibniz Universität Hannover, D-30167 Hannover, Germany \\ ${ }^{5}$ Department of Astronomy and Astrophysics, Penn State University, University Park, Pennsylvania 16802, USA \\ ${ }^{6}$ School of Physics and Astronomy, Cardiff University, Cardiff CF24 3AA, United Kingdom
}

(Received 19 May 2020; revised 7 August 2020; accepted 9 October 2020; published 10 November 2020)

\begin{abstract}
In this Letter, we show that multiband observations of stellar-mass binary black holes by the next generation of ground-based observatories (3G) and the space-based Laser Interferometer Space Antenna (LISA) would facilitate a comprehensive test of general relativity by simultaneously measuring all the post-Newtonian coefficients. Multiband observations would measure most of the known post-Newtonian phasing coefficients to an accuracy below a few percent-2 orders-of-magnitude better than the best bounds achievable from even "golden" binaries in the 3G or LISA bands. Such multiparameter bounds would play a pivotal role in constraining the parameter space of modified theories of gravity beyond general relativity.
\end{abstract}

DOI: 10.1103/PhysRevLett.125.201101

Introduction.-Gravitational wave (GW) observations have provided a first glimpse of the strong-field dynamics of binary black holes (BBHs) [1,2]. They have also allowed us to place the first ever constraints on the possible departures from general relativity (GR) $[3,4]$ in this regime. Parametrized tests of the post-Newtonian (PN) approximation to GR [5-8] are among the most important theoryagnostic, null tests of GR that are performed using GW observations. These tests make use of the analytical prediction of the structure of the phase evolution using the PN approximation to GR [9]. In the PN approximation the dynamics of the binary is treated as an adiabatic process and Einstein's field equations are solved under the assumption of slow motion and weak gravitational fields. This is an excellent approximation for the "inspiral" phase of the compact binary dynamics where the two stars spiralin under the influence of radiation back reaction, but the timescale of radiation reaction is large compared to the orbital timescale.

Gravitational waveform from a compact binary coalescence, in the frequency domain, have the well-known form [10]

$$
\tilde{h}(f)=\mathcal{A} f^{-7 / 6} e^{i \Phi(f)},
$$

where $\Phi(f)$ is the frequency-domain phase of the emitted signal and $\mathcal{A}$ is the signal's amplitude. For inspiraling binaries in quasicircular orbits, the waveform depends on the binary's masses, spins, distance, sky position, and the orientation of its orbit. More explicitly, the phase takes the form

$\Phi(f)=2 \pi f t_{c}-\phi_{c}+\frac{3}{128 \eta v^{5}}\left[\sum_{k=0}^{K} \phi_{k} v^{k}+\sum_{k l=0}^{K} \phi_{k l} v^{k l} \ln v\right]$,

where $v=(\pi m f)^{1 / 3}$ denotes the PN expansion parameter, $m$ denotes the binary's total mass, and $\phi_{k l}$ and $\phi_{k}$ denote the logarithmic and nonlogarithmic phasing coefficients, respectively. The PN coefficients are currently known up to 3.5 order in the PN expansion [11-14], which corresponds to $k=7$ in the above equation. Various PN coefficients capture a range of nonlinear interactions and physical effects in GR [9]. These include the effect of mass asymmetry (1PN and above), different types of "tail" effects (1.5PN, 2.5PN, 3PN, 3.5PN) [15] as well as physical interactions such as spin-orbit (1.5PN, 2.5PN, 3PN, 3.5PN) [16,17] and spin-spin effects (2PN, 3PN) $[16,18]$, and effects due to the presence or absence of a horizon of the compact objects (2.5PN) [19]. (See Ref. [20] for an in-depth discussion about the modifications to the GR phasing formula from various modified theories.) The parameters $t_{c}$ and $\phi_{c}$ are the epoch when the signal's 
amplitude at the detector is the greatest and the phase of the signal at that epoch, respectively. For BBHs on quasicircular orbits, the PN coefficients $\phi_{k}$ and $\phi_{k l}$ are functions of the component masses and spins. The assumption of a quasicircular orbit is an excellent approximation for majority of the stellar-mass BBHs [21].

The parametrized tests rely on the unique prediction for the PN coefficients $\phi_{k}$ and $\phi_{k l}$ in GR and use GW BBH merger events to constrain possible departures of the coefficients from their GR prediction. A parametrized waveform replacing the GR phasing coefficients $\phi_{a}$ with $\phi_{a}\left(1+\delta \hat{\phi}_{a}\right)(a=k, k l)$ is employed for the test [8]. By construction, the deformation parameters $\delta \hat{\phi}_{a}$ are identically equal to zero in GR, while in a modified theory of gravity one or more of these parameters can deviate from zero. Thus, GW data allow the direct measurement of the PN coefficients and if their deformations are found to be consistent with zero, the uncertainty associated with the measurement provides an upper limit on the deviation of these parameters from their GR values.

Status of parametrized tests of post-Newtonian theory.Combining data for the ten $\mathrm{BBH}$ merger events found during the first and second observing runs of LIGO/Virgo, the current bound on the eight PN deformation parameters are given in Fig. 4 of Ref. [4]. Moreover, the bounds from this theory-agnostic test have been mapped onto specific modified theories of gravity in Ref. [20]. However, there is an important caveat while using these bounds to constrain a modified theory of gravity: The bound on the deviation from a particular PN coefficient reported in Ref. [4] is derived assuming that all the deformation parameters except the one that is being tested follow the predictions of GR with $\delta \hat{\phi}_{a}=0$. This assumption is necessary because the most general test wherein all the PN coefficients are simultaneously measured yields very poor or no bounds due to the strong degree of covariance among the deformation parameters and the intrinsic parameters of the binary [22]. Hence, one is compelled to replace this most general test with a series of tests wherein only one deformation parameter is varied at a time together with, of course, the intrinsic parameters of the binary. This restricted suite of tests can still be expected to detect a deviation away from GR by finding statistically significant offsets away from zero in one or more of the PN deformation parameters $[6,8]$.

It would, however, be misleading to use the results from the single-parameter tests to constrain a specific physical effect in a modified theory of gravity. First, any deviation from GR inferred for a particular PN coefficient cannot be attributed uniquely to a breakdown of GR at that PN order. This is because the waveform is largely degenerate in the PN coefficients. Consequently, deviation at a particular PN order can be captured by deformation of the coefficient at a different PN order. Hence, a deviation in one or more of the PN coefficients in a set of tests does not necessarily give any fundamental insight into the true nature of the underlying theory of gravity. Second, if the single-parameter tests are all consistent with GR, the widths of the posterior distributions of the PN coefficients cannot be used to constrain the parameter space of modified theories of gravity. This is due to the expectation from effective field theoretic arguments, that deviations from GR, in a specific modified theory of gravity, show up starting from a certain PN order (see, for instance, [23,24]). Therefore, to map the PN deformation parameters to the free parameters of a specific modified theory of gravity it is necessary to perform the most general multiparameter test. In other words, single-parameter tests would lead to an underestimation of the errors and hence yield bounds that are more stringent than what one might infer with multiparameter tests.

In this Letter, we will show that combining data from the next generation (3G) of ground-based detectors, such as the Cosmic Explorer (CE) [25] and Einstein Telescope (ET) [26], with the space-based LISA observatory [27] is likely the only viable route to carry out this very challenging, but very general test of GR. Such tests are crucial to set reliable constraints on the parameter space of modified theories of gravity. Specifically, we demonstrate that multiband observations of a subclass of stellar-mass BBHs by LISA and CE would provide a unique opportunity to carry out the multiparameter test of PN theory. Combining the lowfrequency sensitivity of LISA with the high-frequency sensitivity of CE helps in lifting the large degeneracies that prevent the use of multiparameter tests in either of these observatories. To demonstrate the advantage of multiparameter tests using multibanding we simulate a stellar-mass population of $\mathrm{BBHs}$ that obey the mass distribution, rate distribution, and redshift distribution inferred from the first and second observing runs of LIGO and Virgo. In a companion paper [28], we will discuss intermediate-mass BBHs as another important class of sources for multiband, multiparameter test of GR, although the bounds from stellar-mass BBHs are far better than their intermediate-mass counterparts [28].

Multiband visibility of stellar-mass binaries.-The planned LISA observatory is sensitive to GWs in the frequency range $\sim 0.1-100 \mathrm{mHz}$ and the proposed $3 \mathrm{G}$ observatories (e.g., CE, which we have used in this Letter as a representative of $3 \mathrm{G}$ detectors), will be sensitive in the frequency range $\sim 1 \mathrm{~Hz}-5 \mathrm{kHz}$. Though LISA is more sensitive to mergers of supermassive $\mathrm{BBHs}$ of millions of solar masses, it has been argued that the detection of stellar-mass BBHs using LISA would be possible despite the small signal-to-noise ratios (SNRs) [29-31] and would be of immense importance to astronomy and fundamental physics, as the mergers of these binaries would be detectable by the ground-based detectors operating at the same time. Observation of sources at earlier stages of their evolution in LISA, and 
later, more nonlinear, stages in $3 \mathrm{G}$ detectors is referred to as multiband observation.

Several authors have investigated the value added by multiband observations of GW sources. For example, Refs. [32-34] examined the projected constraints on the bounds on dipolar GW radiation, Ref. [35] investigated the bounds on single-parameter tests of GR, and Ref. [36] studied the constraints on the parameter space of modified theories of gravity using multiband observations. These authors have used prototypical BBH systems, such as GW150914 [1], which will have good multiband visibility, and have studied the corresponding bounds for singleparameter tests of GR.

Here, we consider $5 \times 10^{5} \mathrm{BBHs}$ corresponding to one year of CE observation [37], distributed uniformly in comoving volume up to redshift $z=10$. The primary black hole masses are assumed to follow a power-law distribution with the power-law index $\alpha=1.6$ [i.e., $\left.p\left(m_{1}\right) \propto m_{1}^{-\alpha}\right]$ in the mass range $[5,100] M_{\odot}$ while secondary masses are uniform in the same mass range [38]. We assume the binary components to possess spins which are aligned or antialigned with respect to the orbital angular momentum vector. This assumption is consistent with the fact that none of the BBHs detected during the first and second observing runs of LIGO/Virgo showed evidence for spins misaligned with the binary's orbital angular momentum. The Kerr parameter of the companion black holes are drawn from two different distributions: (1) a uniform distribution in the range $[0,1]$ and (2) a Gaussian with mean 0 and standard deviation 0.1 .

3G detectors will be able to observe stellar-mass BBH mergers up to the epoch of the formation of first stars. The question is what fraction of events detected by $\mathrm{CE}$ will have LISA counterparts. This joint population will be limited by the SNR in the LISA band. In Fig. 1 we plot the SNR distribution in LISA for this population. As expected, only a small subset of the population will have an SNR greater than 4. Such events will have an SNR of at least 2000 in $\mathrm{CE}$, facilitating a very accurate measurement of the binary parameters, which in turn helps in digging the signals out of the LISA background noise. For example, the chirpmass and the symmetric mass ratio are both measured to an accuracy better than a few parts in a million and the source's position on the sky will be determined to within 75 square arc minutes (enough to identify the host galaxy within $500 \mathrm{Mpc}$ ). Consequently, the number of templates required in archival searches of the LISA data is $<$ few $\times$ $10^{4}$ for $90 \%$ of the events, orders of magnitude smaller than previous estimates [39]. This reduces the false alarm probability of the archival search and makes it possible to identify signals of SNR $>4$ in the LISA data with a false alarm probability of $<10^{-3}$ [40]. We find that among the hundreds of thousands of stellar-mass BBH merger that would be observable by $\mathrm{CE}$ in one year, 200 would cross this threshold of SNR $>4$ and permit multiband,

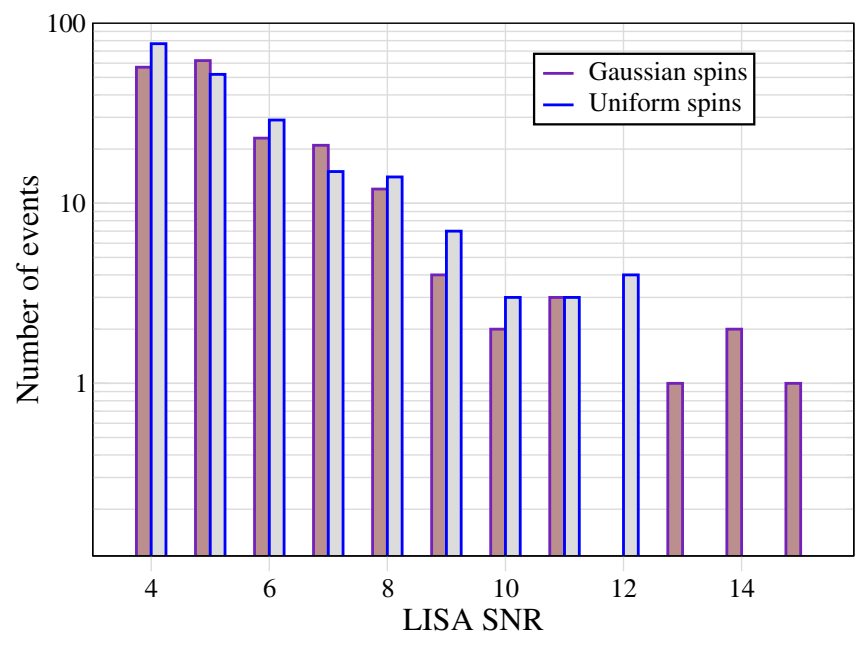

FIG. 1. Distribution of the SNR of stellar-mass BBHs in the LISA band using the mass and redshift distribution as inferred from the first and second observing runs, and spins following (i) a uniform distribution and (ii) a Gaussian distribution with mean 0 and standard deviation 0.1 . Only SNR $\geq 4$ events are shown. A small fraction of about $\sim 200$ of all sources (some 500000 ) observed by $\mathrm{CE}$ in a year have $\mathrm{SNR} \geq 4$ in the LISA band. The plot also shows that the spin distribution of black holes does not have a significant effect on the visibility of stellar-mass BBHs in LISA.

multiparameter tests of GR. These 200 BBHs would spend roughly 4.5 days to 7 weeks outside the LISA band before entering the CE band and eventually merge.

Multiparameter tests of GR via multiband $G W$ observations.-We now describe the efficacy of the multiparameter tests of GR using the population of $\sim 200 \mathrm{BBH}$ merger events detectable by both $\mathrm{CE}$ and LISA. Our method here is based on the well-known Fisher information matrix which enables the computation of the projected $1 \sigma$ errors on the various parameters describing a signal model for a given sensitivity of the detector configuration [45-47]. We use the sensitivity curves of CE and LISA given in [25] and [27], respectively. For simplicity, we do not consider the orbital motion of LISA as it is likely to have negligible impact on the parameter estimation of the intrinsic parameters of the binary, which is of interest here. Stellar-mass BBHs that merge in the CE band are assumed to have been observed for five years in LISA and the starting frequency for the signal in LISA is chosen accordingly following the prescription given in Eq. (2.15) of Ref. [48].

We employ the IMRPhenomD $[49,50]$ waveform model, a frequency-domain phenomenological model describing the complete inspiral-merger-ringdown phases of BBH systems. The waveform amplitude in this model is truncated at the quadrupolar order and we have introduced additional deformation parameters $\delta \hat{\phi}_{a}$ in the phase at different PN orders in the inspiral part of the waveform. We have set the four angles corresponding to the sky position of the binary and the orientation of its orbit with respect to the 
line-of-sight to zero. This amounts to assuming that the binaries are optimally located and oriented with respect to the detectors. Note, however, that the LISA sensitivity curve that we use is averaged over the sky and the polarization angle and we have included a factor of $\sqrt{4 / 5}$ in the calculation of the SNR and the Fisher matrix to account for the averaging over the inclination angle [51].

The Fisher information matrix for a single detector (CE or LISA) is defined as

$$
\Gamma_{\alpha \beta}^{(0)}=\left\langle\tilde{h}_{\alpha}, \tilde{h}_{\beta}\right\rangle,
$$

where $\tilde{h}(f ; \vec{\theta})$ is the GW signal defined by a set of parameters $\vec{\theta}, \tilde{h}_{\alpha}=\partial \tilde{h}(f ; \vec{\theta}) / \partial \theta_{\alpha}$, and the angular bracket $\langle$,$\rangle denotes the noise-weighted inner product defined by$

$$
\langle a, b\rangle=2 \int_{f_{\text {low }}}^{f_{\text {high }}} \frac{a(f) b^{*}(f)+a^{*}(f) b(f)}{S_{h}(f)} d f,
$$

where $S_{h}(f)$ is the one-sided noise power spectral density of the detector and $f_{\text {low }}, f_{\text {high }}$ are the lower and upper limits of integration. For CE the lower limit of integration is taken to be $5 \mathrm{~Hz}$ and the upper frequency cutoff is chosen such that the characteristic amplitude $(2 \sqrt{f}|\tilde{h}(f)|)$ of the GW signal is lower than that of the CE noise by $10 \%$ at maximum.

In order to combine the information from LISA and CE, we construct a multiband Fisher matrix by simply adding the Fisher matrices for the individual detectors, with the corresponding variance-covariance matrix $C^{\alpha \beta}$ defined by the inverse of the multiband Fisher matrix:

$$
\Gamma_{\alpha \beta}=\Gamma_{\alpha \beta}^{\mathrm{CE}}+\Gamma_{\alpha \beta}^{\mathrm{LISA}}, \quad C^{\alpha \beta}=\left(\Gamma^{-1}\right)^{\alpha \beta} .
$$

The diagonal components $C^{\alpha \alpha}$ are the variances of $\theta^{\alpha}$ and the $1 \sigma$ errors on $\theta^{\alpha}$ are $\sigma^{\alpha}=\sqrt{C^{\alpha \alpha}}$.

The errors $\sigma_{a}$, where $a=1,2, \ldots, 8$ denote the deformation parameters that are tested simultaneously, are obtained for each event in the population for different choices of the number of test parameters $\delta \hat{\phi}_{a}$, $a=1,2, \ldots, 8$. The bounds on the individual events are combined to obtain a net constraint by using the standard formula

$$
\sigma_{a}^{-2}=\sum_{n=1}^{N}\left(\sigma_{a}^{(n)}\right)^{-2}
$$

where $n=1, \ldots, N$ denotes the events in the BBH population and $N$ is their total number.

Following Refs. [47,52] we also add a prior matrix $\Gamma^{p}$ to the Fisher information matrix $\Gamma^{(0)}$ in order to account for certain properties of the signals that we assume. Specifically, we assume that the priors on the spin magnitudes and the phase of coalescence as $\Gamma_{\chi_{1} \chi_{1}}^{p}=\Gamma_{\chi_{2} \chi_{2}}^{p}=$ $(0.5)^{-2}$ and $\Gamma_{\phi_{c} \phi_{c}}^{p}=(\pi)^{-2}$, respectively, and all other elements of the prior matrix are set to zero. The Gaussian prior on spin magnitudes is a good approximation to the low-component spins of the BBHs reported in Ref. [2]. The prior on $\phi_{c}$ is somewhat ad hoc, but helps the Fisher matrix to be better conditioned. We have verified that this choice of prior does not alter our conclusions reported in this Letter. We now invert the resulting Fisher matrix given by $\Gamma_{\alpha \beta}=\Gamma_{\alpha \beta}^{0}+\Gamma_{\alpha \beta}^{p}$ to deduce the error bars.

Results and discussions.-Our main results combining LISA and CE observations of stellar-mass BBHs are summarized in Fig. 2. As we increase the number of PN coefficients that are simultaneously tested, starting from the Newtonian order, the $1 \sigma$ upper bounds on them are presented in the figure. For instance, the filled circles are the bounds where only one PN deformation parameter is estimated at a time, whereas the octagons denote the bounds when all the eight parameters are simultaneously estimated. In the eight parameter case, all the parameters are measured with an accuracy $\sim 20 \%$, of which the first three may be measured with an accuracy better than $1 \%$, whereas the first two PN coefficients may yield bounds $\sim 0.1 \%$.

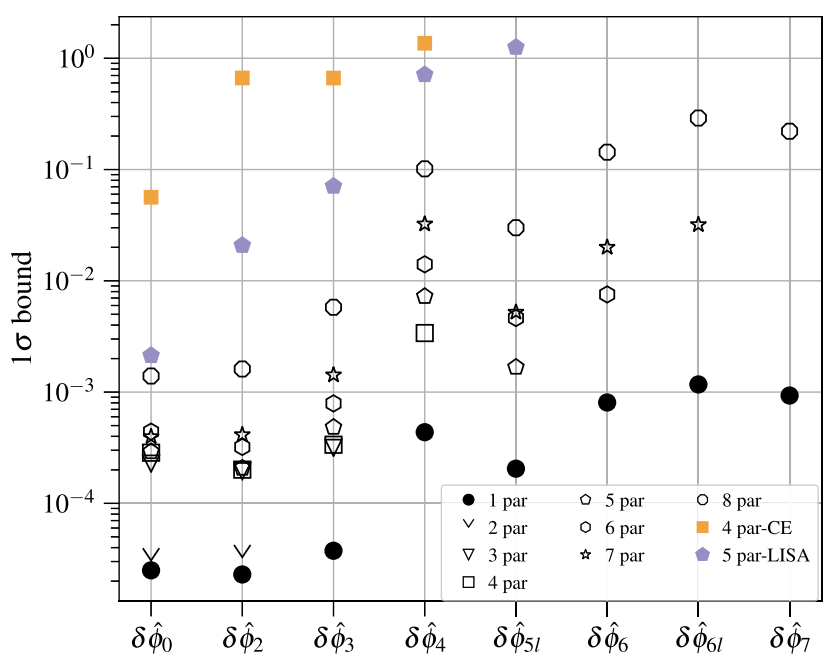

FIG. 2. Multiparameter tests using multiband observations with LISA and CE. Shown are combined $1 \sigma$ bounds on various PN coefficients starting from 0PN through $3.5 \mathrm{PN}$ in the inspiral phase of the signal while measuring many of them together at a time. Different types of markers symbolize how many PN deformation parameters were constrained simultaneously. For example, "black filled circle" represents "one PN deformation parameter at a time," "vee" represents "two PN deformation parameters at a time," and so on. The figure represents results for the $\mathrm{BBH}$ population having Gaussian spin distribution, we get similar estimates for a uniform spin distribution. The filled diamonds and pentagons are bounds obtained with $\mathrm{CE}$ and LISA, respectively, on the first four and five PN deformation parameters from their respective golden binaries, respectively. The total masses of the CE and LISA golden binaries are $200 M_{\odot}$ and $\left(6.6 \times 10^{5}\right) M_{\odot}$, both binaries are $1 \mathrm{Gpc}$ away and have component spins $\chi_{1}=0.6, \chi_{2}=0.5$. 
One may notice interesting trends in the bounds as we add more and more parameters. The bounds on OPN and 1PN deformation coefficient from 2-parameter estimation case are $<0.01 \%$. The inclusion of the $1.5 \mathrm{PN}$ deformation coefficient results in a sudden worsening of the bounds by an order of magnitude. This may be understood by noting that $1.5 \mathrm{PN}$ is the order at which spins first appear in the phasing formula. Adding a deformation parameter at that order, that is completely degenerate with spins, adversely affects the overall parameter estimation, which gets reflected in the bounds on the first two PN coefficients. The gradual worsening of the bounds as we go to even higher multiparameter tests is simply due to the increasing degeneracy brought in by each of the additional PN deformation parameters. Nevertheless, multiband observations of stellar-mass BBHs would permit us to test modified theories of gravity, which predict deviations at orders below $3 \mathrm{PN}$ to a precision less than $1 \%$.

It can be seen that even in the era of $3 \mathrm{G}$ detectors we cannot obtain meaningful constraints with multiparameter tests. As is evident from Fig. 2, for golden binaries in CEbinaries that have the smallest error for the multiparameter tests - the errors on $\delta \phi_{a}$, are $\sim 100 \%$ only for $a=1, \ldots, 4$; if we vary more than four parameters at a time then the errors on PN coefficients with $a \neq 0$ are larger than $100 \%$. In a year's time CE will observe a handful of such golden binaries and the joint error that one can obtain by combining golden binaries will still not be significantly smaller. Consequently, ground-based detectors alone cannot break the degeneracy among different PN coefficients. The same is true with LISA observations of supermassive BBHs. Even with a golden supermassive BBH we can perform the multiparameter test with only five parameters and LISA is not likely to observe more than a handful of such binaries over a five-year period. Having said this, in this Letter we do not consider other ways to compute the combined bounds on $\delta \phi_{a}$, such as by combining all the events observed in CE and LISA, as our method already achieves the desired accuracy needed for the multiparameter test.

Conclusions.-To conclude, we have shown the importance of multiband observations of GWs to carry out the multiparameter tests of GR. From our systematic study of a representative set of systems, we have also found that even for the best case scenario, observations of supermassive BBHs in the LISA band or stellar- or intermediate-mass BBHs in the CE band would not be able to place constraints as good as the one reported here. Hence, multibanding would, perhaps, be the only way to carry out this test which in turn is necessary to make meaningful constraints on the parameter space of modified theories of gravity. As LIGO and Virgo detect several more BBHs in the future observing runs, the merger rate and the mass distribution would be more tightly constrained, which is likely to further tighten the bounds derived here making this test an excellent science case for multiband observations.
We thank Chris Van Den Broeck, Bala Iyer, Arnab Dhani, and M. Saleem for several useful discussions. B. S. S. is supported in part by NSF Grants No. PHY-1836779, No. AST-1716394, and No. AST-1708146. S. B. is supported by NSF Grant No. PHY-1836779. K. G. A. and S.D. are partially supported by a grant from the Infosys Foundation. They also acknowledge the Swarnajayanti Grant No. DST/SJF/PSA-01/2017-18 DST-India. K. G. A acknowledges Core Research Grant No. EMR/2016/005594 of SERB. We acknowledge the use of IUCAA LDG cluster Sarathi for the computational and numerical work.

*agupta1@olemiss.edu

[1] B. P. Abbott et al. (Virgo, LIGO Scientific Collaborations), Phys. Rev. Lett. 116, 061102 (2016).

[2] B. P. Abbott et al. (LIGO Scientific, Virgo Collaborations), Phys. Rev. X 9, 031040 (2019).

[3] B. P. Abbott et al. (Virgo, LIGO Scientific Collaborations), Phys. Rev. Lett. 116, 221101 (2016).

[4] B. P. Abbott et al. (LIGO Scientific, Virgo Collaborations), Phys. Rev. D 100, 104036 (2019).

[5] K. G. Arun, B. R. Iyer, M. S. S. Qusailah, and B. S. Sathyaprakash, Phys. Rev. D 74, 024006 (2006).

[6] C. K. Mishra, K. G. Arun, B. R. Iyer, and B. S. Sathyaprakash, Phys. Rev. D 82, 064010 (2010).

[7] N. Yunes and F. Pretorius, Phys. Rev. D 80, 122003 (2009).

[8] T. G. F. Li et al., Phys. Rev. D 85, 082003 (2012); J. Phys. Conf. Ser. 363, 012028 (2012).

[9] L. Blanchet, Living Rev. Relativity 9, 4 (2006); 17, 2 (2014).

[10] B. S. Sathyaprakash and S. V. Dhurandhar, Phys. Rev. D 44, 3819 (1991).

[11] L. Blanchet, T. Damour, B. R. Iyer, C. M. Will, and A. G. Wiseman, Phys. Rev. Lett. 74, 3515 (1995).

[12] L. Blanchet, T. Damour, G. Esposito-Farèse, and B. R. Iyer, Phys. Rev. Lett. 93, 091101 (2004).

[13] K. G. Arun, A. Buonanno, G. Faye, and E. Ochsner, Phys. Rev. D 79, 104023 (2009).

[14] C. K. Mishra, A. Kela, K. G. Arun, and G. Faye, Phys. Rev. D 93, 084054 (2016).

[15] L. Blanchet and T. Damour, Phys. Rev. D 37, 1410 (1988).

[16] L. E. Kidder, C. M. Will, and A. G. Wiseman, Phys. Rev. D 47, R4183 (1993).

[17] L. Blanchet, A. Buonanno, and G. Faye, Phys. Rev. D 74, 104034 (2006); 75, 049903(E) (2007).

[18] A. Buonanno, G. Faye, and T. Hinderer, Phys. Rev. D 87, 044009 (2013).

[19] H. Tagoshi, S. Mano, and E. Takasugi, Prog. Theor. Phys. 98, 829 (1997).

[20] N. Yunes, K. Yagi, and F. Pretorius, Phys. Rev. D 94, 084002 (2016).

[21] A. Buonanno, B. R. Iyer, E. Ochsner, Y. Pan, and B. S. Sathyaprakash, Phys. Rev. D 80, 084043 (2009).

[22] K. G. Arun, B. R. Iyer, M. S. S. Qusailah, and B. S. Sathyaprakash, Classical Quantum Gravity 23, L37 (2006).

[23] S. Endlich, V. Gorbenko, J. Huang, and L. Senatore, J. High Energy Phys. 09 (2017) 122. 
[24] N. Sennett, R. Brito, A. Buonanno, V. Gorbenko, and L. Senatore, Phys. Rev. D 102, 044056 (2020).

[25] D. Reitze et al., Bull. Am. Astron. Soc. 51, 035 (2019), https://baas.aas.org/pub/2020n7i035/release/1.

[26] M. Punturo et al., Classical Quantum Gravity 27, 194002 (2010).

[27] P. Amaro-Seoane, H. Audley, S. Babak, J. Baker, E. Barausse, P. Bender, E. Berti, P. Binetruy, M. Born, and D. Bortoluzzi, arXiv:1702.00786.

[28] S. Datta, A. Gupta, S. Kastha, K. Arun, and B. Sathyaprakash, arXiv:2006.12137.

[29] R. Nair, S. Jhingan, and T. Tanaka, Prog. Theor. Exp. Phys. 2016, 053E01 (2016).

[30] S. Vitale, Phys. Rev. Lett. 117, 051102 (2016).

[31] A. Sesana, Phys. Rev. Lett. 116, 231102 (2016).

[32] E. Barausse, N. Yunes, and K. Chamberlain, Phys. Rev. Lett. 116, 241104 (2016).

[33] A. Toubiana, S. Marsat, S. Babak, E. Barausse, and J. Baker, Phys. Rev. D 101, 104038 (2020).

[34] C. Liu, L. Shao, J. Zhao, and Y. Gao, Mon. Not. R. Astron. Soc. 496, 182 (2020).

[35] Z. Carson and K. Yagi, Classical Quantum Gravity 37, 02LT01 (2020).

[36] G. Gnocchi, A. Maselli, T. Abdelsalhin, N. Giacobbo, and M. Mapelli, Phys. Rev. D 100, 064024 (2019).

[37] V. Baibhav, E. Berti, D. Gerosa, M. Mapelli, N. Giacobbo, Y. Bouffanais, and U.N. Di Carlo, Phys. Rev. D 100, 064060 (2019).

[38] B. P. Abbott et al. (LIGO Scientific, Virgo Collaborations), Astrophys. J. 882, L24 (2019).
[39] K. W. K. Wong, E. D. Kovetz, C. Cutler, and E. Berti, Phys. Rev. Lett. 121, 251102 (2018).

[40] See Supplemental Material at http://link.aps.org/ supplemental/10.1103/PhysRevLett.125.201101 for a discussion on the detectability of stellar-mass BBH signals in LISA with an SNR threshold as low as 4, which includes Refs. [41-44].

[41] C. J. Moore, D. Gerosa, and A. Klein, Mon. Not. R. Astron. Soc. 488, L94 (2019).

[42] B. J. Owen and B. Sathyaprakash, Phys. Rev. D 60, 022002 (1999).

[43] K. Cannon et al., Astrophys. J. 748, 136 (2012).

[44] N. Yunes and S. A. Hughes, Phys. Rev. D 82, 082002 (2010).

[45] C. Rao, Bull. Calcutta Math. Soc. 37, 81 (1945).

[46] H. Cramer, Mathematical Methods in Statistics (Pergamon Press, Princeton University Press, NJ, 1946).

[47] C. Cutler and E. E. Flanagan, Phys. Rev. D 49, 2658 (1994).

[48] E. Berti, A. Buonanno, and C. M. Will, Phys. Rev. D 71, 084025 (2005).

[49] S. Husa, S. Khan, M. Hannam, M. Pürrer, F. Ohme, X. J. Forteza, and A. Bohé, Phys. Rev. D 93, 044006 (2016).

[50] S. Khan, S. Husa, M. Hannam, F. Ohme, M. Pürrer, X. J. Forteza, and A. Bohé, Phys. Rev. D 93, 044007 (2016).

[51] T. Robson, N. J. Cornish, and C. Liu, Classical Quantum Gravity 36, 105011 (2019).

[52] E. Poisson and C. M. Will, Phys. Rev. D 52, 848 (1995). 